\title{
QUADRATURE AND SYMMETRY ON THE CUBED SPHERE
}

\author{
JEAN-BAPTISTE BELLET ${ }^{\dagger}$, MATTHIEU BRACHET $^{\ddagger}$, AND JEAN-PIERRE CROISILLE $^{\dagger}$
}

\begin{abstract}
In the companion paper [6], a spherical harmonics subspace associated to the Cubed Sphere has been introduced. This subspace is further analyzed here. In particular, it permits to define a new Cubed Sphere based quadrature. This quadrature inherits the rotationally invariant properties of the spherical harmonics subspace. Contrary to Gauss quadratures, where the set of nodes and weights is solution of a nonlinear system, only the weights are unknown here. Despite this conceptual simplicity, the new quadrature displays an accuracy comparable to optimal quadratures, such as the Lebedev rules.
\end{abstract}

\section{INTRODUCTION}

We introduce a new quadrature rule defined on the equiangular Cubed Sphere grid.

Let $\mathrm{CS}_{N} \subset \mathbb{S}^{2}$ denote the Cubed Sphere with parameter $N$. In [6], a finite dimensional subspace of spherical harmonics has been associated to the set of nodes $\mathrm{CS}_{N}$. This subspace, called $\mathcal{U}_{N}$ herefater, provides existence and uniqueness to the Lagrange interpolation, with $\mathrm{CS}_{N}$ as set of nodes. Here our goal is to use the space $\mathcal{U}_{N}$ to define a new quadrature rule on the sphere $\mathbb{S}^{2}$, which uses $\mathrm{CS}_{N}$ as set of nodes.

Previous studies have considered the construction of Gauss quadratures on the sphere. In this case, the set of nodes and the associated weights are both unknown. Establishing the set of equations for the nodes and the weights is difficult. The modern analysis of this approach relies on the theory of t-designs, whose main purpose is to find an optimal distribution of nodes on the sphere. This theory has a mathematical and physical interest in itself. We refer to $[9]$ for a review. The identification of a quadrature associated to a particular t-design is obtained as byproduct in a second time. Once the quadrature nodes are known, the weights are commonly indentified by requiring exactness for a set of spherical harmonics. A general approach is to select this set as all spherical harmonics of degree smaller than a given value.

Here the Cubed Sphere nodes are selected from the beginning as "good" quadrature nodes, and therefore, only the weights have to be identified. In [15], two examples of weights have been suggested. The first one was based on some extended trapezoidal rule, attributing some area to each node. The second one was thought as a perturbation with a design based on some optimization principle. See also [7] for some other rules, including some Simpson formula. Here we come back to the general question of the "best choice" of weights associated to the Cubed Sphere nodes. As in the general approach, we require exactness of the quadrature for a particular set of spherical harmonics. Using the space $\mathcal{U}_{N}$ in $\left.\mid 6\right]$ immediately delivers a quadrature rule. This quadrature is different from the ones mentionned above. The space $\mathcal{U}_{N}$ remarkably enjoys invariance under the action of the group of the cube. This is somehow expected, since the group of $\mathrm{CS}_{N}$ is in fact the group of the cube, (or of the octahedron) [5]. As will be shown below, the new quadrature rule inherits this invariance. This property is highly desirable. It is well known that group invariance is the backbone for the design of highly accurate spherical quadratures, $[1,13,14,17]$. Refer for this to the review [12].

The paper is organized as follows. Section 2 gives the notation on the spherical harmonics, the Cubed Sphere and its group. Section 3 recalls how is defined the space $\mathcal{U}_{N}$ and Section 4 establishes several of its invariance properties. In Section 5, the new quadrature is introduced. By construction, this quadrature is exact on the space $\mathcal{U}_{N}$. In addition, it is invariant under the octahedral group. This in particular implies that it is exact for a proportion of 15/16 of all (real) Legendre spherical harmonics. Finally in Section 6, we display numerical results for a large series of test cases. It is observed that the new rule is only slightly suboptimal, when compared to the optimal Lebedev rules. This somehow supports the main Ansatz of this study, namely that the Cubed Sphere nodes are good quadrature nodes on the sphere. Finally we report some comments on the accuracy of the new rule in Appendix A.

Date: May 10, 2021.

2010 Mathematics Subject Classification. 65D05, 65D30, 65D32.

Key words and phrases. Cubed sphere, interpolation, quadrature, octahedral group. 


\section{BACKGROUND AND NOTATION}

The real Legendre spherical harmonics of degree $n$ are normalized as follows:

$$
Y_{n}^{m}(x)=Y_{n}^{m}(\theta, \phi)=(-1)^{|m|} \sqrt{\frac{(n+1 / 2)(n-|m|) !}{\pi(n+|m|) !}} P_{n}^{|m|}(\sin \theta) \times \begin{cases}\sin |m| \phi, & m<0 \\ \frac{1}{\sqrt{2}}, & m=0 \\ \cos m \phi, & m>0\end{cases}
$$

Here, $P_{n}^{|m|}(t)=(-1)^{|m|}\left(1-t^{2}\right)^{|m| / 2} \frac{\mathrm{d}^{|m|+n}}{\mathrm{~d} t^{|m|+n}} \frac{1}{2^{n} n !}\left(t^{2}-1\right)^{n}$ is the associated Legendre function, and we identify $x=(\cos \theta \cos \phi, \cos \theta \sin \phi, \sin \theta)$ with $(\theta, \phi) ; \phi \in[-\pi, \pi]$ is the longitude, $\theta \in\left[-\frac{\pi}{2}, \frac{\pi}{2}\right]$ is the latitude. The family $\left(Y_{n}^{m}\right)_{-n \leq m \leq n}$ is an orthonormal basis of the subspace $\mathcal{Y}_{n} \subset L^{2}\left(\mathbb{S}^{2}\right)$, for the inner product

$$
\langle u, v\rangle_{L^{2}\left(\mathbb{S}^{2}\right)}=\int_{\mathbb{S}^{2}} u(x) v(x) \mathrm{d} \sigma .
$$

The infinite family $\left(Y_{n}^{m}\right)_{|m| \leq n, n \in \mathbb{N}}$ is a Hilbert basis of $L^{2}\left(\mathbb{S}^{2}\right)$.

The equiangular Cubed Sphere $\mathrm{CS}_{N} \subset \mathbb{S}^{2}$, is defined by

$$
\begin{aligned}
\mathrm{CS}_{N}:=\left\{\frac{1}{\sqrt{1+u^{2}+v^{2}}}( \pm 1, u, v), \frac{1}{\sqrt{1+u^{2}+v^{2}}}(u, \pm 1, v), \frac{1}{\sqrt{1+u^{2}+v^{2}}}(u, v, \pm 1) ;\right. \\
\left.u=\tan \frac{i \pi}{2 N}, v=\tan \frac{j \pi}{2 N},-\frac{N}{2} \leq i, j \leq \frac{N}{2}\right\} .
\end{aligned}
$$

For ease of notation, we denote the nodes of $\mathrm{CS}_{N}$ by $x_{i}, 1 \leq i \leq \bar{N}$, where $\bar{N}=6 N^{2}+2$ is the cardinal number:

$$
\mathrm{CS}_{N}=\left\{x_{i}, 1 \leq i \leq \bar{N}\right\}
$$

The symmetry group of the Cubed Sphere is given in the following theorem [5].

Theorem 1 (Symmetry group of the Cubed Sphere). An orthogonal matrix $Q$ leaves the Cubed Sphere $\mathrm{CS}_{N}$ invariant if, and only if, it leaves the octahedron

$$
\{( \pm 1,0,0),(0, \pm 1,0),(0,0, \pm 1)\}
$$

invariant. In other words, the symmetry group $\mathcal{G}$ of $\mathrm{CS}_{N}$ is exactly the full octahedral group. Its matrix representation is given by

$$
\mathcal{G}=\left\{\left[\begin{array}{lll}
\epsilon_{1} e_{\sigma_{1}} & \epsilon_{2} e_{\sigma_{2}} & \epsilon_{3} e_{\sigma_{3}}
\end{array}\right], \sigma \in \mathfrak{S}_{3}, \epsilon \in\{-1,1\}^{3}\right\}, \quad \text { with } \quad e_{1}=(1,0,0), e_{2}=(0,1,0), e_{3}=(0,0,1) .
$$

The space of real functions defined on $\mathrm{CS}_{N}$ is denoted by

$$
\mathcal{F}\left(\mathrm{CS}_{N}\right)=\left\{f: \mathrm{CS}_{N} \rightarrow \mathbb{R}\right\} .
$$

The canonical basis $\left(\delta_{x_{i}}\right)_{1 \leq i \leq \bar{N}}$ of $\mathcal{F}\left(\mathrm{CS}_{N}\right)$ is defined by

$$
\delta_{x_{i}}\left(x_{j}\right)=\delta_{i j}=\left\{\begin{array}{ll}
1, & \text { if } i=j, \\
0, & \text { otherwise, }
\end{array} \quad 1 \leq i, j \leq \bar{N} .\right.
$$

In this basis, any $f \in \mathcal{F}\left(\mathrm{CS}_{N}\right)$ is represented by the column vector $\left[f\left(x_{i}\right)\right]_{1 \leq i \leq \bar{N}} \in \mathbb{R}^{\bar{N}}$, due to the decomposition

$$
f=\sum_{i=1}^{\bar{N}} f\left(x_{i}\right) \delta_{x_{i}}
$$

For any real function defined on the sphere, $u: x \in \mathbb{S}^{2} \mapsto u(x) \in \mathbb{R}$, the restriction of $u$ on $\operatorname{CS}_{N}$ is the function defined by

$$
\left.u\right|_{\mathrm{CS}_{N}}:=\sum_{i=1}^{\bar{N}} u\left(x_{i}\right) \delta_{x_{i}} \in \mathcal{F}\left(\mathrm{CS}_{N}\right),\left.\quad u\right|_{\mathrm{CS}_{N}}\left(x_{i}\right)=u\left(x_{i}\right), 1 \leq i \leq \bar{N} .
$$

\section{Interpolation on the Cubed Sphere with spherical harmonics}

In [6], we have introduced a subspace $\mathcal{U}_{N}$ of real spherical harmonics, dedicated to Lagrange interpolation on the Cubed Sphere $\mathrm{CS}_{N}$. Our presentation here is deduced from intrinsic properties of $\mathcal{U}_{N}$ proved in 6$]^{1}$

First, we introduce a subspace $\mathcal{W}_{n}$ of spherical harmonics of degree $n$, defined by

$$
\mathcal{W}_{0}:=\{0\}, \quad \mathcal{W}_{n}:=\left\{u \in \mathcal{Y}_{n}: \exists v \in \mathcal{Y}_{0} \oplus \cdots \oplus \mathcal{Y}_{n-1},\left.u\right|_{\mathrm{CS}_{N}}=\left.v\right|_{\mathrm{CS}_{N}}\right\}, n \geq 1
$$

\footnotetext{
${ }^{1}$ The space $\mathcal{U}_{N}$ was denoted by $\mathcal{Y}_{N^{\prime}}^{\prime}$ in $[6]$.
} 
Intuitively, $\mathcal{W}_{n}$ contains spherical harmonics of degree $n$ which are undersampled on $\mathrm{CS}_{N}$. Second, we build an orthogonal decomposition based on this subspace,

$$
\mathcal{Y}_{n}=\mathcal{V}_{n} \oplus \mathcal{W}_{n}, \quad \text { with } \quad \mathcal{V}_{n}:=\left(\mathcal{W}_{n}\right)^{\perp \mathcal{Y}_{n}}=\left\{u \in \mathcal{Y}_{n}: \forall v \in \mathcal{W}_{n},\langle u, v\rangle_{L^{2}\left(\mathbb{S}^{2}\right)}=0\right\}
$$

In the orthogonal complement $\mathcal{V}_{n}$, any spherical harmonics can be reconstructed by interpolation on $\mathrm{CS}_{N}$ (unlike $\mathcal{W}_{n}$ ). This claim is part of the following theorem [6].

Theorem 2. There exists an integer $N^{\prime}$ such that the Lagrange interpolation problem has always a unique solution in $\mathcal{V}_{0} \oplus \cdots \oplus \mathcal{V}_{N^{\prime}}$, i.e.

$$
\forall f \in \mathcal{F}\left(\mathrm{CS}_{N}\right), \quad \exists ! u \in \mathcal{V}_{0} \oplus \cdots \oplus \mathcal{V}_{N^{\prime}},\left.\quad u\right|_{\mathrm{CS}_{N}}=f, \quad\left(\text { i.e. }, \forall 1 \leq j \leq \bar{N}, u\left(x_{j}\right)=f\left(x_{j}\right)\right) .
$$

This theorem justifies the following definition.

Definition 3 (Interpolation space). Let $\mathcal{V}_{n}$ denote the orthogonal complement $(3)$ of the undersampled subspace $\mathcal{W}_{n}$, defined by (2). The interpolation space on $\mathrm{CS}_{N}$ is defined as

$$
\mathcal{U}_{N}:=\mathcal{V}_{0} \oplus \cdots \oplus \mathcal{V}_{N^{\prime}}
$$

where $N^{\prime} \geq 0$ is the smallest integer such that (4) is realized.

We refer to [6] for a practical algorithm to compute a real orthonormal basis of $\mathcal{U}_{N}$, denoted here by $\left(u_{j}\right)_{1 \leq j \leq \bar{N}}$. Then,

$$
u_{j} \in \mathcal{U}_{N}, \quad\left\langle u_{i}, u_{j}\right\rangle_{L^{2}\left(\mathbb{S}^{2}\right)}=\delta_{i j}, \quad 1 \leq i, j \leq \bar{N} .
$$

Without loss of generality, the first basis function is $u_{1}(x)=\frac{1}{\sqrt{4 \pi}} \in \mathcal{Y}_{0}$. By definition of $\mathcal{U}_{N}$, the following linear map is isomorphic:

$$
\begin{aligned}
T_{N}: \mathcal{U}_{N} & \longrightarrow \mathcal{F}\left(\mathrm{CS}_{N}\right) \\
u & \left.\longmapsto u\right|_{\mathrm{CS}_{N}} .
\end{aligned}
$$

The matrix of $T_{N}$, in the canonical basis $\left(\delta_{x_{i}}\right)_{1 \leq i \leq \bar{N}}$ of $\mathcal{F}\left(\mathrm{CS}_{N}\right)$, and the basis $\left(u_{j}\right)_{1 \leq j \leq \bar{N}}$ of $\mathcal{U}_{N}$, is the Vandermonde matrix given by

$$
A=\left[u_{j}\left(x_{i}\right)\right]_{1 \leq i, j \leq \bar{N}}
$$

where $i$ is the row index, and $j$ is the column index. In (4), the solution $u \in \mathcal{U}_{N}$ is given by $u=T_{N}^{-1} f \in \mathcal{U}_{N}$. This suggests to define an interpolation operator, by

$$
\mathcal{I}_{N}:=T_{N}^{-1} \text {. }
$$

Then the unique element of $\mathcal{U}_{N}$ interpolating $f \in \mathcal{F}\left(\mathrm{CS}_{N}\right)$ is given by $\mathcal{I}_{N} f \in \mathcal{U}_{N}$, and is represented by the column vector $A^{-1}\left[f\left(x_{i}\right)\right] \in \mathbb{R}^{\bar{N}}$,

$$
\mathcal{I}_{N} f(x)=\left[u_{j}(x)\right] A^{-1}\left[f\left(x_{i}\right)\right], \quad x \in \mathbb{S}^{2} .
$$

\section{Rotational invariance of the interpolation SPACE}

In this section, we study the invariance of the interpolation space $\mathcal{U}_{N}$ under the symmetry group $\mathcal{G}$ of the Cubed Sphere. We call "rotated" a function defined as follows.

Definition 4 ("Rotated" function). Assume that $Q \in \mathcal{G}$ leaves a set $E$ invariant, i.e. $Q^{\top} E=E$. Let $f: E \rightarrow \mathbb{R}$ be a function defined on $E$. The "rotated" function, denoted by $f\left(Q^{\top} \cdot\right)$, is the function

$$
f\left(Q^{\top} \cdot\right): x \in E \mapsto f\left(Q^{\top} x\right) \in \mathbb{R} .
$$

Our main invariance result is the following theorem.

Theorem 5 (Invariance of the interpolation space). Let $n \geq 0$.

(i) The undersampled subspace $\mathcal{W}_{n}$ is invariant under $\mathcal{G}$, i.e.

$$
\forall Q \in \mathcal{G}, \forall u \in \mathcal{W}_{n}, u\left(Q^{\top} \cdot\right) \in \mathcal{W}_{n} .
$$

(ii) The subspace $\mathcal{V}_{n}=\mathcal{W}_{n}^{\perp}$ is invariant under $\mathcal{G}$, i.e.

$$
\forall Q \in \mathcal{G}, \forall u \in \mathcal{V}_{n}, u\left(Q^{\top} \cdot\right) \in \mathcal{V}_{n} .
$$

(iii) The interpolation space $\mathcal{U}_{N}$ is invariant under $\mathcal{G}$, i.e.

$$
\forall Q \in \mathcal{G}, \forall u \in \mathcal{U}_{N}, u\left(Q^{\top} \cdot\right) \in \mathcal{U}_{N} .
$$

Proof. Fix $Q \in \mathcal{G}$, i.e. $Q \in \mathbb{R}^{3 \times 3}$ is an orthogonal matrix such that $Q^{\top} \mathrm{CS}_{N}=\mathrm{CS}_{N}$.

(i) If $n=0, \mathcal{W}_{0}=\{0\}$ is invariant under $\mathcal{G}$. Fix now $u \in \mathcal{W}_{n} \subset \mathcal{Y}_{n}$ with $n \geq 1$. There exists $v \in \mathcal{Y}_{0} \oplus \cdots \oplus \mathcal{Y}_{n-1}$ such that $\left.u\right|_{\mathrm{CS}_{N}}=\left.v\right|_{\mathrm{CS}_{N}}$, or equivalently, $\left.(u-v)\right|_{\mathrm{CS}_{N}}=0$. Firstly, $u\left(Q^{\top} \cdot\right) \in \mathcal{Y}_{n}$ and $v\left(Q^{\top} \cdot\right) \in \mathcal{Y}_{0} \oplus \cdots \oplus \mathcal{Y}_{n-1}$. Secondly, $\left.\left(u\left(Q^{\top} \cdot\right)-v\left(Q^{\top} \cdot\right)\right)\right|_{\mathrm{CS}_{N}}=\left.(u-v)\right|_{\mathrm{CS}_{N}}\left(Q^{\top} \cdot\right)=0$, and therefore $\left.u\left(Q^{\top} \cdot\right)\right|_{\mathrm{CS}_{N}}=\left.v\left(Q^{\top} \cdot\right)\right|_{\mathrm{CS}_{N}}$; here, the commutation between rotation and restriction is justified by the following lemma. 
Lemma 6 (Rotation commutes with restriction). For all $Q \in \mathcal{G}, n \geq 0$, and $u \in \mathcal{Y}_{0} \oplus \cdots \oplus \mathcal{Y}_{n}$,

$$
\left.u\left(Q^{\top} \cdot\right)\right|_{\mathrm{CS}_{N}}=\left.u\right|_{\mathrm{CS}_{N}}\left(Q^{\top} \cdot\right) \in \mathcal{F}\left(\mathrm{CS}_{N}\right) \text {. }
$$

We postpone the proof of the lemma until the end of this section.

(ii) The result is a combination of (i) and $\mathcal{V}_{n}=\mathcal{W}_{n}^{\perp}$. Indeed, fix $u \in \mathcal{V}_{n} \subset \mathcal{Y}_{n}$ with $n \geq 0$. Then $u\left(Q^{\top}\right.$.) $\in \mathcal{Y}_{n}$. Furthermore, for every $v \in \mathcal{W}_{n}$,

$$
\left\langle u\left(Q^{\top} \cdot\right), v\right\rangle_{L^{2}\left(\mathbb{S}^{2}\right)}=\int_{\mathbb{S}^{2}} u\left(Q^{\top} x\right) v(x) \mathrm{d} \sigma=\int_{\mathbb{S}^{2}} u(y) v(Q y) \mathrm{d} \sigma=\langle u, v(Q \cdot)\rangle_{L^{2}\left(\mathbb{S}^{2}\right)} ; \quad\left(y:=Q^{\top} x\right) .
$$

$\mathcal{W}_{n}$ is invariant under $\mathcal{G}$, so $v(Q \cdot) \in \mathcal{W}_{n}$. Then $v(Q \cdot)$ is orthogonal to $u$ because $u \in \mathcal{W}_{n}^{\perp}$. We obtain $\left\langle u\left(Q^{\top} \cdot\right), v\right\rangle=\langle u, v(Q \cdot)\rangle=0$, which proves $u\left(Q^{\top} \cdot\right) \in \mathcal{W}_{n}^{\perp}$.

(iii) The space $\mathcal{U}_{N}=\mathcal{V}_{0} \oplus \cdots \oplus \mathcal{V}_{N^{\prime}}$ is a sum of invariant subspaces due to (ii).

Corollary 7 (Interpolation and symmetry). (i) The interpolation operator commutes with any symmetry of the group $\mathcal{G}$ :

$$
\forall f \in \mathcal{F}\left(\mathrm{CS}_{N}\right), \forall Q \in \mathcal{G},\left[\mathcal{I}_{N} f\right]\left(Q^{\top} \cdot\right)=\mathcal{I}_{N}\left[f\left(Q^{\top} \cdot\right)\right] .
$$

(ii) The interpolation operator preserves the invariance property; in other words, if $f \in \mathcal{F}\left(\mathrm{CS}_{N}\right)$ is invariant under $\mathcal{G}$, i.e. $\forall Q \in \mathcal{G}, f\left(Q^{\top} \cdot\right)=f$, then $\mathcal{I}_{N} f$ is invariant under $\mathcal{G}$, i.e. $\forall Q \in \mathcal{G},\left[\mathcal{I}_{N} f\right]\left(Q^{\top} \cdot\right)=\mathcal{I}_{N} f$.

Proof. (i) Firstly, $f\left(Q^{\top} \cdot\right) \in \mathcal{F}\left(\mathrm{CS}_{N}\right)$ and $u=\mathcal{I}_{N}\left[f\left(Q^{\top} \cdot\right)\right] \in \mathcal{U}_{N}$ is the unique element of $\mathcal{U}_{N}$ such that $\left.u\right|_{\mathrm{CS}_{N}}=f\left(Q^{\top}\right.$.). Secondly, $v=\mathcal{I}_{N} f \in \mathcal{U}_{N}$ is the unique element of $\mathcal{U}_{N}$ such that $\left.v\right|_{\mathrm{CS}_{N}}=f$. Due to Theorem 5. (iii), $v\left(Q^{\top} \cdot\right) \in \mathcal{U}_{N}$. By Lemma 6, $\left.v\left(Q^{\top} \cdot\right)\right|_{\mathrm{CS}_{N}}=\left.v\right|_{\mathrm{CS}_{N}}\left(Q^{\top} \cdot\right)=f\left(Q^{\top}\right.$.), which proves $u=v\left(Q^{\top}\right.$.). (ii) is an immediate consequence of (i).

Proof of Lemma 6. Firstly, $\mathcal{Y}_{0} \oplus \cdots \oplus \mathcal{Y}_{n}$ is left invariant by $Q$, so $\left[u\left(Q^{\top} \cdot\right): x \in \mathbb{S}^{2} \mapsto u\left(Q^{\top} x\right)\right] \in \mathcal{Y}_{0} \oplus \cdots \oplus \mathcal{Y}_{n}$, and $\left.u\left(Q^{\top} \cdot\right)\right|_{\mathrm{CS}_{N}}$ is defined by

$$
\left.u\left(Q^{\top} \cdot\right)\right|_{\mathrm{CS}_{N}}=\sum_{i=1}^{\bar{N}} u\left(Q^{\top} x_{i}\right) \delta_{x_{i}} \in \mathcal{F}\left(\mathrm{CS}_{N}\right) .
$$

On the other hand, $\left[\left.u\right|_{\mathrm{CS}_{N}}: x \in \mathrm{CS}_{N} \mapsto u(x)\right] \in \mathcal{F}\left(\mathrm{CS}_{N}\right)$, with $\mathrm{CS}_{N}$ left invariant by $Q$. Then the function $\left.u\right|_{\mathrm{CS}_{N}}\left(Q^{\top} \cdot\right)$ is well-defined and is given by

$$
\left.u\right|_{\mathrm{CS}_{N}}\left(Q^{\top} \cdot\right): x \in \mathrm{CS}_{N} \mapsto u\left(Q^{\top} x\right) \in \mathcal{F}\left(\mathrm{CS}_{N}\right) .
$$

At every $x=x_{i} \in \mathrm{CS}_{N}$, the two functions have the same value, $u\left(Q^{\top} x_{i}\right)$.

\section{A new quadrature on the Cubed Sphere}

In this section, we study a new quadrature rule on $\mathrm{CS}_{N}$; it is defined by interpolation as follows.

Theorem 8 (Quadrature rule). Let $u: \mathbb{S}^{2} \rightarrow \mathbb{R}$ be a given function. The quadrature rule $\mathcal{Q}_{N}$ is defined by

$$
\mathcal{Q}_{N} u:=\int_{\mathbb{S}^{2}} \mathcal{I}_{N}\left[\left.u\right|_{\mathrm{CS}_{N}}\right](x) \mathrm{d} \sigma .
$$

(i) The formula $\mathcal{Q}_{N}$ can be written as follows:

$$
\mathcal{Q}_{N} u=\sum_{j=1}^{\bar{N}} \omega_{N}\left(x_{j}\right) u\left(x_{j}\right), \quad \text { with } \omega_{N} \in \mathcal{F}\left(\mathrm{CS}_{N}\right) \text { such that }\left[\omega_{N}\left(x_{j}\right)\right]=\left(A^{\top}\right)^{-1}\left[\begin{array}{llll}
\sqrt{4 \pi} & 0 & \cdots & 0
\end{array}\right]^{\top} ;
$$

here, $A$ is the Vandermonde matrix (5).

(ii) The formula $\mathcal{Q}_{N}$ is exact on $\mathcal{U}_{N}$, i.e.

$$
\forall u \in \mathcal{U}_{N}, \quad \mathcal{Q}_{N} u=\int_{\mathbb{S}^{2}} u(x) \mathrm{d} \sigma .
$$

(iii) The rule $\mathcal{Q}_{N}$ and the weight $\omega_{N}$ are invariant under $\mathcal{G}$, i.e.

$$
\forall Q \in \mathcal{G}, \quad \forall u \in \mathcal{U}_{N}, \mathcal{Q}_{N}\left(u\left(Q^{\top} \cdot\right)\right)=\mathcal{Q}_{N}(u), \quad \text { and } \omega_{N}\left(Q^{\top} \cdot\right)=\omega_{N} .
$$

Proof. (i-ii) Firstly, if $u \in \mathcal{U}_{N}, \mathcal{Q}_{N}$ exactly integrates $u$, since $u$ coincides with $\mathcal{I}_{N} u$. In particular, for each basis function $u_{j} \in \mathcal{U}_{N}, \mathcal{Q}_{N} u_{j}=\int_{\mathbb{S}^{2}} u_{j}(x) \mathrm{d} \sigma$. For $u_{1}=\frac{1}{\sqrt{4 \pi}}, \int_{\mathbb{S}^{2}} u_{1}(x) \mathrm{d} \sigma=\sqrt{4 \pi}$. For every $1 \leq j \leq \bar{N}$, $u_{j} \perp u_{1}$, which means $\int_{\mathbb{S}^{2}} u_{j}(x) \mathrm{d} \sigma=0$. Then, $\left[\mathcal{Q}_{N} u_{j}\right]_{1 \leq j \leq \bar{N}}=[\sqrt{4 \pi} 0 \cdots 0]^{\top}$. Secondly, fix $\omega_{N} \in \mathcal{F}\left(\mathrm{CS}_{N}\right)$ such that

$$
\omega_{N}\left(x_{i}\right)=\int_{\mathbb{S}^{2}} \mathcal{I}_{N}\left[\delta_{x_{i}}\right] \mathrm{d} \sigma, \quad 1 \leq i \leq \bar{N} .
$$


By linearity, we deduce from (1) that

$$
\mathcal{Q}_{N} u=\sum_{i=1}^{\bar{N}} \omega_{N}\left(x_{i}\right) u\left(x_{i}\right)=\left[u\left(x_{i}\right)\right]^{\top}\left[\omega_{N}\left(x_{i}\right)\right] .
$$

Using the basis functions, we obtain

$$
A^{\top}\left[\omega_{N}\left(x_{i}\right)\right]_{1 \leq i \leq \bar{N}}=\left[\mathcal{Q}_{N} u_{j}\right]_{1 \leq j \leq \bar{N}}=\left[\begin{array}{llll}
\sqrt{4 \pi} & 0 & \cdots & 0
\end{array}\right]^{\top},
$$

where the matrix $A^{\top} \in \mathbb{R}^{\bar{N} \times \bar{N}}$ is non singular.

Remark 9. In [6], a LQ-factorization of $A^{\top}$ is computed, $A^{\top}=L Q$ where $L$ is lower triangular, whereas $Q$ is orthogonal. So, $\omega_{N}$ can be deduced from a linear system whose matrix is $L:\left[\omega_{N}\left(x_{j}\right)\right]=Q^{\top} L^{-1}\left[\begin{array}{llll}4 \pi & 0 & \cdots\end{array}\right]^{\top}$.

(iii) Fix $Q \in \mathcal{G}$ and $u \in \mathcal{U}_{N}$. By Theorem 5, $u\left(Q^{\top} \cdot\right) \in \mathcal{U}_{N}$. Thus, by (ii) and change of variable,

$$
\mathcal{Q}_{N}\left(u\left(Q^{\top} \cdot\right)\right)=\int_{\mathbb{S}^{2}} u\left(Q^{\top} x\right) \mathrm{d} \sigma=\int_{\mathbb{S}^{2}} u(x) \mathrm{d} \sigma=\mathcal{Q}_{N}(u) \quad\left(x:=Q^{\top} x\right) .
$$

Fix now $1 \leq i \leq \bar{N}$ and $u=\mathcal{I}_{N} \delta_{x_{i}} \in \mathcal{U}_{N}$. By Corollary $7, u\left(Q^{\top} \cdot\right)=\mathcal{I}_{N}\left[\delta_{x_{i}}\left(Q^{\top} \cdot\right)\right] \in \mathcal{U}_{N}$, with $\delta_{x_{i}}\left(Q^{\top}\right.$. $)=\delta_{Q x_{i}}$. Therefore by (7), $\mathcal{Q}_{N}(u)=\omega_{N}\left(x_{i}\right)$ and $\mathcal{Q}_{N}\left(u\left(Q^{\top} \cdot\right)\right)=\omega_{N}\left(Q x_{i}\right)$. Then, by invariance of $\mathcal{Q}_{N}$,

$$
\omega_{N}\left(x_{i}\right)=\mathcal{Q}_{N}(u)=\mathcal{Q}_{N}\left(u\left(Q^{\top} \cdot\right)\right)=\omega_{N}\left(Q x_{i}\right) .
$$

The quadrature rule exactly integrates the $\bar{N}$ spherical harmonics of $\mathcal{U}_{N}$. Taking benefit from the rotational invariance, we prove furthermore that it exactly integrates an infinite number of spherical harmonics.

Corollary 10. The quadrature rule $\mathcal{Q}_{N}$ exactly integrates $\frac{15}{16}$ of all real Legendre spherical harmonics. More precisely, for all $|m| \leq n$,

$$
\mathcal{Q}_{N}\left(Y_{n}^{m}\right)=\int_{\mathbb{S}^{2}} Y_{n}^{m}(x) \mathrm{d} \sigma, \quad \text { if } \quad\left\{\begin{array}{l}
n \equiv 1(2) \\
m<0, \\
m \geq 0 \text { and } m \equiv 1,2,3(4) ;
\end{array}\right.
$$

equivalently, $\mathcal{Q}_{N}\left(Y_{n}^{m}\right) \neq \int_{\mathbb{S}^{2}} Y_{n}^{m}(x) \mathrm{d} \sigma \Rightarrow n \equiv 0(2), m \geq 0$ and $m \equiv 0$ (4).

Proof. Fix $n \geq 1$ and $|m| \leq n$. Then $\int_{\mathbb{S}^{2}} Y_{n}^{m}(x) \mathrm{d} \sigma=0$. For well chosen $n, m$, we build a symmetry $Q \in \mathcal{G}$ such that $Y_{n}^{m}\left(Q^{\top} \cdot\right)=-Y_{n}^{m}$. In such cases, we obtain $\mathcal{Q}_{N}\left(Y_{n}^{m}\right)=\mathcal{Q}_{N}\left(Y_{n}^{m}\left(Q^{\top} \cdot\right)\right)=-\mathcal{Q}_{N}\left(Y_{n}^{m}\right)$, which proves $\mathcal{Q}_{N}\left(Y_{n}^{m}\right)=0=\int_{\mathbb{S}^{2}} Y_{n}^{m}(x) \mathrm{d} \sigma$. Recall that $x(\theta, \phi)=(\cos \theta \cos \phi, \cos \theta \sin \phi, \sin \theta), \phi \in[-\pi, \pi], \theta \in\left[-\frac{\pi}{2}, \frac{\pi}{2}\right]$, and $Y_{n}^{m}(x(\theta, \phi)):=Y_{n}^{m}(\theta, \phi)$.

Case 1: $n \equiv 1(2)$ and $m \equiv 0(4)$. Then $\theta \mapsto P_{n}^{|m|}(\sin \theta)$ is odd, so is $\theta \mapsto Y_{n}^{m}(x(\theta, \phi))$; hence,

$$
Y_{n}^{m}\left(Q^{\top} x(\theta, \phi)\right)=Y_{n}^{m}(x(-\theta, \phi))=-Y_{n}^{m}(x(\theta, \phi)), \quad \text { for } \quad Q:=\left[\begin{array}{ccc}
1 & 0 & 0 \\
0 & 1 & 0 \\
0 & 0 & -1
\end{array}\right] \text {. }
$$

Case 2: $m<0$. Then $\phi \mapsto Y_{n}^{m}(x(\theta, \phi))$ is odd, so,

$$
Y_{n}^{m}\left(Q^{\top} x(\theta, \phi)\right)=Y_{n}^{m}(x(\theta,-\phi))=-Y_{n}^{m}(x(\theta, \phi)), \quad \text { for } \quad Q:=\left[\begin{array}{ccc}
1 & 0 & 0 \\
0 & -1 & 0 \\
0 & 0 & 1
\end{array}\right] .
$$

Case 3: $m \equiv 1,3(4)$. Then $m(\phi+\pi) \equiv m \phi+\pi(2 \pi)$, and

$$
Y_{n}^{m}\left(Q^{\top} x(\theta, \phi)\right)=Y_{n}^{m}(x(\theta, \phi+\pi))=-Y_{n}^{m}(x(\theta, \phi)), \quad \text { for } \quad Q:=\left[\begin{array}{ccc}
-1 & 0 & 0 \\
0 & -1 & 0 \\
0 & 0 & 1
\end{array}\right] \text {. }
$$

Case 4: $m \equiv 2(4)$. Then $m\left(\phi+\frac{\pi}{2}\right) \equiv m \phi+\pi(2 \pi)$, and

$$
Y_{n}^{m}\left(Q^{\top} x(\theta, \phi)\right)=Y_{n}^{m}\left(x\left(\theta, \phi+\frac{\pi}{2}\right)\right)=-Y_{n}^{m}(x(\theta, \phi)), \quad \text { for } \quad Q:=\left[\begin{array}{ccc}
0 & 1 & 0 \\
-1 & 0 & 0 \\
0 & 0 & 1
\end{array}\right] \text {. }
$$

Remark 11. The ratio 15/16 of the real Legendre basis is asymptotic. In [7, 15], a similar approach based on invariance properties provided an asymptotic ratio of $7 / 8$ of the complex Legendre basis. We observe here that using the real Legendre basis highlights further exact results. 


\section{NumericAl RESUlts}

6.1. Symmetry invariance assessment. We begin by two numerical assessments related to interpolation in $\mathcal{U}_{N}$.

First, we illustrate that the interpolation operator $\mathcal{I}_{N}$ preserves the invariance property, as stated in Corollary 7.(ii). Fix $N=6$ and consider the series of symmetric functions $g_{i} \in \mathcal{F}\left(\mathrm{CS}_{N}\right)$, described in Table 1. By construction, each function $g_{i}, 1 \leq i \leq 6$, is constant along any orbit, i.e. $\forall Q \in \mathcal{G}, g_{i}\left(Q^{\top}\right.$. $)=g_{i}$, and is supported by a set of symmetric nodes. For $i \leq 5, g_{i}$ takes the value 1 along the orbit of $a_{i} \in \mathrm{CS}_{N}$, and the value 0 otherwise. The orbit of $a_{1}$ contains the vertices of an octahedron. The orbit of $a_{2}$ contains the vertices of a cube. The orbit of $a_{3}$ contains the vertices of a cubaoctahedron. The orbit of $a_{4}$ is included in the edges of an octahedron. The orbit of $a_{5}$ is "generic", with cardinal number 48. In Figure1, we visualize how the symmetry is reflected in the interpolating functions $\mathcal{I}_{N} g_{i} \in \mathcal{U}_{N}, 1 \leq i \leq 6$. The octahedral symmetry predicted by Corollary 7 (ii) can be observed; the functions $\mathcal{I}_{N} g_{i}$ are constant along any orbit.

\begin{tabular}{|c|c|c|c|}
\hline$i$ & $g_{i}$ & $a_{i}$ & $\left|\operatorname{supp} g_{i}\right|$ \\
\hline 1 & $\frac{1}{8} \sum_{Q \in \mathcal{G}} \delta_{Q a_{1}}$ & {$\left[\begin{array}{lll}1 & 0 & 0\end{array}\right]^{\top}$} & 6 \\
\hline 2 & $\frac{1}{6} \sum_{Q \in \mathcal{G}} \delta_{Q a_{2}}$ & $\frac{1}{\sqrt{3}}\left[\begin{array}{lll}1 & 1 & 1\end{array}\right]^{\top}$ & 8 \\
\hline 3 & $\frac{1}{4} \sum_{Q \in \mathcal{G}} \delta_{Q a_{3}}$ & $\frac{1}{\sqrt{2}}\left[\begin{array}{lll}1 & 0 & 1\end{array}\right]^{\top}$ & 12 \\
\hline 4 & $\frac{1}{2} \sum_{Q \in \mathcal{G}} \delta_{Q a_{4}}$ & $\left(1+\tan ^{2} \frac{\pi}{6}\right)^{-1 / 2}\left[10 \tan \frac{\pi}{6}\right]^{\top}$ & 24 \\
\hline 5 & $\sum_{Q \in \mathcal{G}} \delta_{Q a_{5}}$ & $\left(1+\tan ^{2} \frac{\pi}{12}+\tan ^{2} \frac{\pi}{6}\right)^{-1 / 2}\left[1 \tan \frac{\pi}{12} \tan \frac{\pi}{6}\right]^{\top}$ & 48 \\
\hline 6 & $g_{1}+g_{2}+g_{3}+g_{4}+g_{5}$ & & 98 \\
\hline
\end{tabular}

TABLE 1. Grid functions with octahedral symmetry. The function $g_{i}, 1 \leq i \leq 6$, takes the value 1 on its support and is invariant under $\mathcal{G}$.
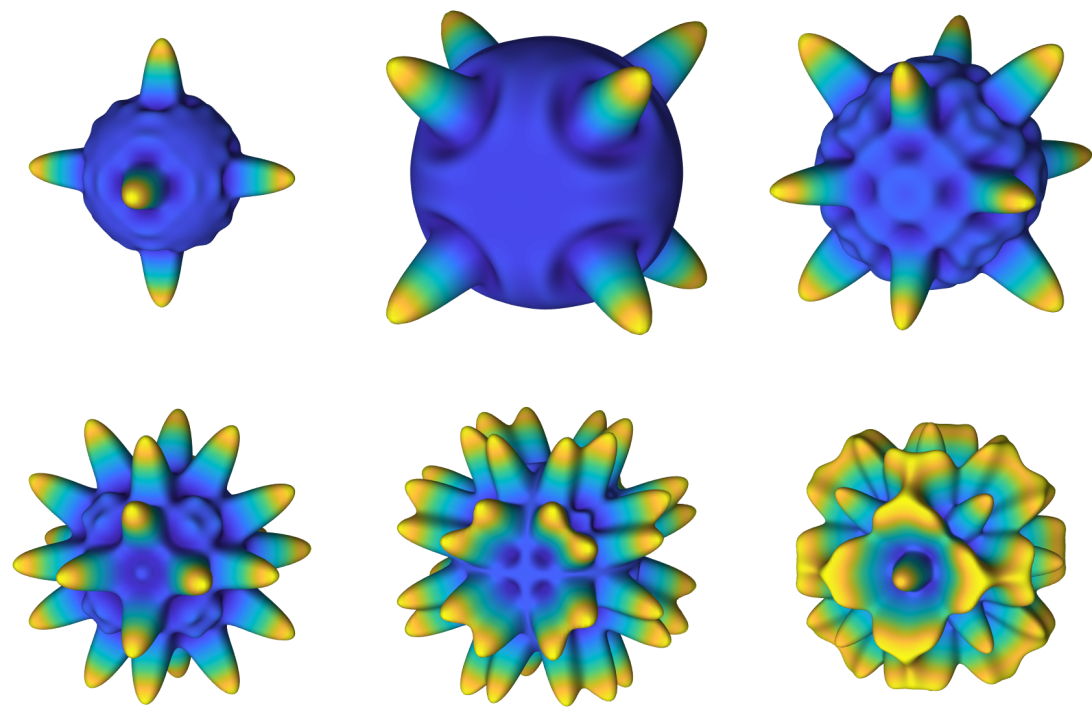

FiguRE 1. Interpolation with octahedral symmetry. For every $1 \leq i \leq 6$, the symmetric function $\mathcal{I}_{N} g_{i}$ is represented by the surface $\left(1.5+\mathcal{I}_{N} g_{i}(x)\right) x, x \in \mathbb{S}^{2}$.

Second, we assess the invariance of the interpolation space, stated in Theorem 5, and the commutation between interpolation and rotation, stated in Corollary 7.(i). For that purpose, for each basis function $u_{j} \in \mathcal{U}_{N}$, we compare $u_{j}\left(Q^{\top} \cdot\right)=\left[\mathcal{I}_{N} u_{j}\right]\left(Q^{\top} \cdot\right)$ with $\mathcal{I}_{N}\left[u_{j}\left(Q^{\top} \cdot\right)\right]$. Indeed, by linearity, Corollary 7 . (i) is equivalent to:

$$
\forall 1 \leq j \leq \bar{N}, \forall Q \in \mathcal{G},\left[\mathcal{I}_{N} u_{j}\right]\left(Q^{\top} \cdot\right)=\mathcal{I}_{N}\left[u_{j}\left(Q^{\top} \cdot\right)\right] .
$$

If this condition is achieved, then for each basis function $u_{j}, u_{j}\left(Q^{\top} \cdot\right)=\left[\mathcal{I}_{N} u_{j}\right]\left(Q^{\top} \cdot\right) \in \operatorname{Ran} \mathcal{I}_{N}=\mathcal{U}_{N}$, which implies Theorem 5.(iii) by linearity. This also implies Theorem 5.(ii), due to $u_{j}\left(Q^{\top}\right.$.) $\in \mathcal{Y}_{n} \cap \mathcal{U}_{N}=\mathcal{V}_{n}$. We compare the functions on a fine grid $\mathrm{CS}_{M}(M=33)$, by computing the relative error

$$
\epsilon_{N, j}(Q):=\frac{\max _{x \in \mathrm{CS}_{M}}\left|u_{j}\left(Q^{\top} x\right)-\mathcal{I}_{N}\left[u_{j}\left(Q^{\top} \cdot\right)\right](x)\right|}{\max _{x \in \mathrm{CS}_{M}}\left|u_{j}(x)\right|} .
$$


Then we compute the maximal error $\epsilon_{N}$, and we repeat the procedure for several values of $N$ :

$$
\epsilon_{N}:=\max \left\{\epsilon_{N, j}(Q), Q \in \mathcal{G}, 1 \leq j \leq \bar{N}\right\}, \quad 1 \leq N \leq 16 .
$$

The results reported in Table 2 are in agreement with the invariance stated in Theorem 5 and Corollary 7 (i).

\begin{tabular}{c|cccccccc}
\hline$N$ & 1 & 2 & 3 & 4 & 5 & 6 & 7 & 8 \\
$\epsilon_{N}$ & $2.5 \mathrm{e}-15$ & $3.4 \mathrm{e}-15$ & $7.8 \mathrm{e}-15$ & $1.4 \mathrm{e}-14$ & $9.7 \mathrm{e}-15$ & $9.3 \mathrm{e}-15$ & $1.3 \mathrm{e}-14$ & $1.1 \mathrm{e}-14$ \\
\hline$N$ & 9 & 10 & 11 & 12 & 13 & 14 & 15 & 16 \\
$\epsilon_{N}$ & $1.4 \mathrm{e}-14$ & $1.5 \mathrm{e}-14$ & $1.9 \mathrm{e}-14$ & $1.7 \mathrm{e}-14$ & $3.4 \mathrm{e}-14$ & $2.2 \mathrm{e}-14$ & $1.8 \mathrm{e}-14$ & $2.9 \mathrm{e}-14$ \\
\hline
\end{tabular}

TABle 2. Numerical invariance: $u_{j}\left(Q^{\top} \cdot\right)=\mathcal{I}_{N}\left[u_{j}\left(Q^{\top} \cdot\right)\right], Q \in \mathcal{G}$, up to relative error $\epsilon_{N}$ (8).

6.2. Quadrature weight. We have computed the quadrature weight $\omega_{N} \in \mathcal{F}\left(\mathrm{CS}_{N}\right)$ for $1 \leq N \leq 32$, and $N=64$. Some of them are displayed in Figure 2, As can been observed, the weight is positive, $\omega_{N}>0$, and the maximum value is reached at the center of a panel. Moreover, some statistics are diplayed in Figure 3. They reveal that the weight $\omega_{N}$ is quasi-uniform. In particular,

$$
\frac{\max \omega_{N}}{\min \omega_{N}} \approx \sqrt{2}
$$

We recognize the ratio between the surface element at the center of a panel of $\mathrm{CS}_{N}$, and the smallest surface element of a panel, as it is derived in [16, Eq. (20)].

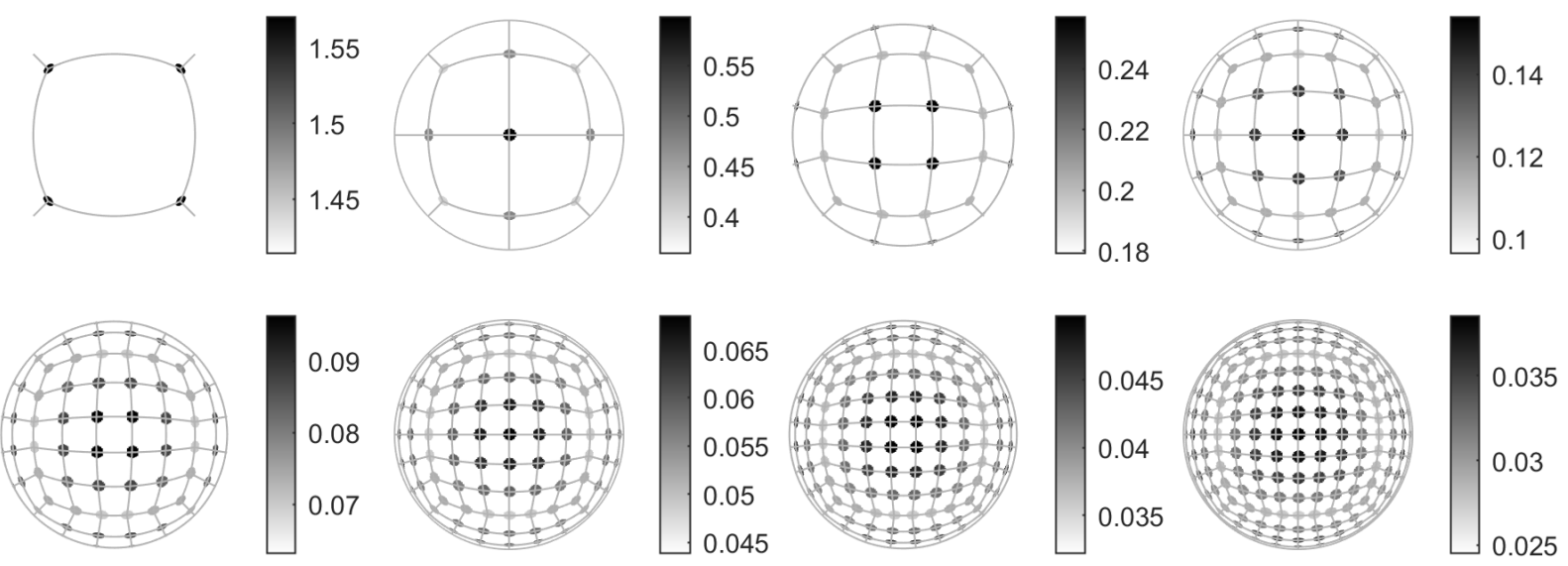

FiguRE 2. Quadrature weight $\omega_{N}$, for $1 \leq N \leq 8$.

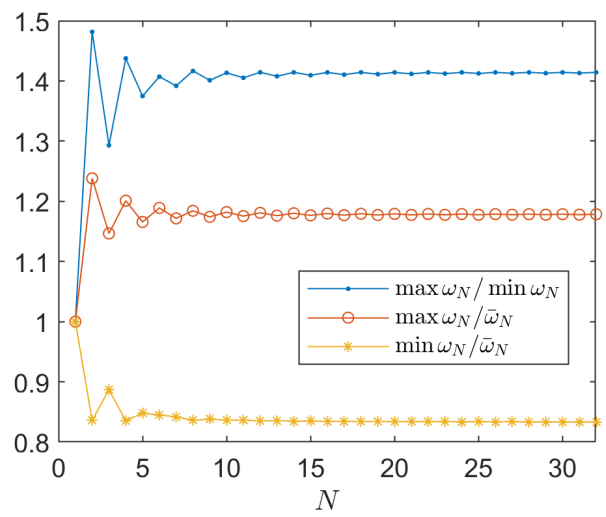

Figure 3. Statistical distribution of the quadrature weight $\omega_{N}$ in $(6), 1 \leq N \leq 32$. The maximum, minimum, and mean values satisfy $\max \omega_{N} \approx 1.41 \min \omega_{N}, \max \omega_{N} \approx 1.18 \bar{\omega}_{N}$, and $\min \omega_{N} \approx 0.83 \bar{\omega}_{N}$. 


\begin{tabular}{|c|c|c|c|}
\hline$i$ & $f_{i}(x, y, z)$ & $\int_{\mathbb{S}^{2}} f_{i}(x, y, z) \mathrm{d} \sigma$ & Ref. \\
\hline 1 & $\exp (x)$ & $14.7680137457653 \cdots$ & \begin{tabular}{|l|l|}
3 & 10 \\
\end{tabular} \\
\hline 2 & $\begin{array}{l}\frac{3}{4} \exp \left[-\frac{(9 x-2)^{2}}{4}-\frac{(9 y-2)^{2}}{4}-\frac{(9 z-2)^{2}}{4}\right] \\
\quad+\frac{3}{4} \exp \left[-\frac{(9 x+1)^{2}}{49}-\frac{9 y+1}{10}-\frac{9 z+1}{10}\right] \\
\quad+\frac{1}{2} \exp \left[-\frac{(9 x-7)^{2}}{4}-\frac{(9 y-3)^{2}}{4}-\frac{(9 z-5)^{2}}{4}\right] \\
\quad-\frac{1}{5} \exp \left[-(9 x-4)^{2}-(9 y-7)^{2}-(9 z-5)^{2}\right]\end{array}$ & $6.6961822200736179523 \ldots$ &  \\
\hline 3 & $\frac{1}{10} \frac{\exp (x+2 y+3 z)}{\left(x^{2}+y^{2}+(z+1)^{2}\right)^{1 / 2}} \mathbf{1}(z>-1)$ & $4.090220018862976 \cdots$ & 2 \\
\hline 4 & $\cos (3 \arccos z) \mathbf{1}\left(3 \arccos z \leq \frac{\pi}{2}\right)$ & $\frac{\pi}{8}$ & inspired from $[2]$ \\
\hline 5 & $\mathbf{1}\left(z \geq \frac{1}{2}\right)$ & $\frac{8}{\pi}$ & \\
\hline 6 & $\frac{1}{9}[1+\operatorname{sign}(-9 x-9 y+9 z)]$ & $\frac{4 \pi}{9}$ & \begin{tabular}{|l|l|l|}
$4,7,11,15$ \\
\end{tabular} \\
\hline
\end{tabular}

TABLE 3 . Test functions and exact integration values.



Figure 4. Test functions of Table 3

6.3. Quadrature of test functions. We test the accuracy of the quadrature formula $\mathcal{Q}_{N}$ on the series of functions reported in Table 3. They are displayed in Figure 4. Most of these functions serve as testing functions for quadrature assessment. References are indicated in Table 3 . The exponential function $f_{1}$ is a smooth, non trivial function. The Franke function $f_{2}$ is a standard test case. The function $f_{3}$ is smooth, except near the South pole, where it has an infinite spike. The cosine cap function $f_{4}$ is continuous but is not differentiable on the circle $z=\frac{\sqrt{3}}{2}$. The function $f_{5}$ is the characteristic function of a spherical cap; it is not continuous. Similarly, the discontinuous function $f_{6}$ represents an hemisphere; it is a standard test function.

We report in Table 4 the quadrature error

$$
\eta_{N}\left(f_{i}\right)=\left|\int_{\mathbb{S}^{2}} f_{i} \mathrm{~d} \sigma-\mathcal{Q}_{N} f_{i}\right|, \quad N=1,2,4,8,16,32,64, \quad 1 \leq i \leq 6 .
$$

Moreover, Table 5 reports a rate of convergence $r_{N}\left(f_{i}\right)$, defined by the equation

$$
\eta_{2 N}\left(f_{i}\right)=\frac{\eta_{N}\left(f_{i}\right)}{2^{r_{N}}\left(f_{i}\right)}
$$

Note that the computations have been performed with Matlab, in double precision. In particular the machine epsilon is approximately $2.2 \times 10^{-16}$; we do not compute the rate when the relative error is close to this value. For the smooth function $f_{1}$, the error rapidly reaches a value which is about $10^{-14}$. For the Franke function $f_{2}$, a thinner grid is required to reach such values, but a very fast convergence is still observed. For the spike function $f_{3}$, the convergence rate is $r_{N}\left(f_{3}\right) \approx 1$. For the continous cap function $f_{4}$, and the discontinuous one $f_{5}$, the error slowly decrease, at a convergence rate which depends on the grid size. For the cap function $f_{6}$, which is discontinous and "symmetric" (supported by a hemisphere), the error is close to the machine epsilon, independently of the grid size. 


\begin{tabular}{rr|cccccc}
\hline$N$ & $\bar{N}$ & $\eta_{N}\left(f_{1}\right)$ & $\eta_{N}\left(f_{2}\right)$ & $\eta_{N}\left(f_{3}\right)$ & $\eta_{N}\left(f_{4}\right)$ & $\eta_{N}\left(f_{5}\right)$ & $\eta_{N}\left(f_{6}\right)$ \\
\hline 1 & 8 & $4.8 \mathrm{e}-02$ & $8.2 \mathrm{e}-01$ & $2.4 \mathrm{e}-01$ & $3.9 \mathrm{e}-01$ & $3.1 \mathrm{e}+00$ & $6.7 \mathrm{e}-16$ \\
2 & 26 & $2.0 \mathrm{e}-06$ & $1.5 \mathrm{e}-02$ & $1.7 \mathrm{e}-02$ & $2.1 \mathrm{e}-01$ & $9.9 \mathrm{e}-01$ & $0.0 \mathrm{e}+00$ \\
4 & 98 & $1.2 \mathrm{e}-14$ & $2.2 \mathrm{e}-03$ & $7.8 \mathrm{e}-03$ & $2.0 \mathrm{e}-02$ & $6.7 \mathrm{e}-02$ & $2.2 \mathrm{e}-16$ \\
8 & 386 & $1.8 \mathrm{e}-15$ & $9.0 \mathrm{e}-06$ & $3.8 \mathrm{e}-03$ & $4.8 \mathrm{e}-03$ & $6.4 \mathrm{e}-02$ & $6.7 \mathrm{e}-16$ \\
16 & 1538 & $7.1 \mathrm{e}-15$ & $5.5 \mathrm{e}-09$ & $1.9 \mathrm{e}-03$ & $3.0 \mathrm{e}-04$ & $1.5 \mathrm{e}-02$ & $6.7 \mathrm{e}-16$ \\
32 & 6146 & $5.3 \mathrm{e}-15$ & $1.8 \mathrm{e}-15$ & $9.5 \mathrm{e}-04$ & $3.1 \mathrm{e}-04$ & $7.9 \mathrm{e}-03$ & $6.7 \mathrm{e}-16$ \\
64 & 24578 & $3.6 \mathrm{e}-15$ & $1.8 \mathrm{e}-15$ & $4.8 \mathrm{e}-04$ & $1.9 \mathrm{e}-07$ & $2.6 \mathrm{e}-03$ & $4.4 \mathrm{e}-16$ \\
\hline
\end{tabular}

TABLE 4. Quadrature error $\eta_{N}\left(f_{i}\right)=\left|\int_{\mathbb{S}^{2}} f_{i} \mathrm{~d} \sigma-\mathcal{Q}_{N} f_{i}\right|$.

\begin{tabular}{r|ccccc|ccccc}
\hline$N$ & $r_{N}\left(f_{1}\right)$ & $r_{N}\left(f_{2}\right)$ & $r_{N}\left(f_{3}\right)$ & $r_{N}\left(f_{4}\right)$ & $r_{N}\left(f_{5}\right)$ & $\bar{r}_{N}\left(f_{1}\right)$ & $\bar{r}_{N}\left(f_{2}\right)$ & $\bar{r}_{N}\left(f_{3}\right)$ & $\bar{r}_{N}\left(f_{4}\right)$ & $\bar{r}_{N}\left(f_{5}\right)$ \\
\hline 1 & 15 & 5.8 & 3.9 & 0.93 & 1.7 & 15 & 2.8 & 5.4 & 2.5 & -0.0033 \\
2 & 27 & 2.7 & 1.1 & 3.4 & 3.9 & 26 & 3.3 & 1.1 & 3.3 & 1.8 \\
4 & 2.8 & 7.9 & 1 & 2.1 & 0.077 & 2.1 & 4.1 & 1 & 1.9 & 1.3 \\
8 & & 11 & 1 & 4 & 2.1 & & 13 & 1.2 & 2.8 & 1.7 \\
16 & & 22 & 1 & -0.047 & 0.92 & & 24 & 0.92 & 2.2 & 1.4 \\
32 & & & 1 & 11 & 1.6 & & & 0.93 & 2.7 & 1.6 \\
\hline
\end{tabular}

TABLE 5. Convergence rate $r_{N}\left(f_{i}\right)$ of the error $\eta_{N}\left(f_{i}\right)$, and convergence rate $\bar{r}_{N}\left(f_{i}\right)$ of the average error $\bar{\epsilon}_{N}\left(f_{i}\right)$, over 1000 random orthogonal transformations of the grid.

6.4. Sensitivity to the grid orientation. Here we consider more closely the accuracy of the rule $\mathcal{Q}_{N}$ : we modify randomly the orientation of the grid, [11,15]. We compute

$$
\epsilon_{N}\left(f_{i}, Q\right)=\left|\int_{\mathbb{S}^{2}} f_{i} \mathrm{~d} \sigma-\mathcal{Q}_{N} f_{i}\left(Q^{\top} \cdot\right)\right|,
$$

where $Q$ browses a set of 1000 randomly selected orthogonal matrices (uniform law in $[0,2 \pi]$ for the Euler angles, and uniform law in $\{-1,1\}$ for the orientation). The worst error, the average error and their ratio are defined by

$$
\varepsilon_{N}\left(f_{i}\right)=\max _{Q} \epsilon_{N}\left(f_{i}, Q\right), \quad \bar{\epsilon}_{N}\left(f_{i}\right)=\frac{1}{1000} \sum_{Q} \epsilon_{N}\left(f_{i}, Q\right), \quad \rho_{N}\left(f_{i}\right)=\frac{\bar{\epsilon}_{N}\left(f_{i}\right)}{\varepsilon_{N}\left(f_{i}\right)}
$$

The worst error $\varepsilon_{N}\left(f_{i}\right)$ and the ratio $\rho_{N}\left(f_{i}\right)$ are displayed ${ }^{2}$ in Figure 5 . We report in Table 5 a convergence rate $\bar{r}_{N}\left(f_{i}\right)$ of the average error $\bar{\epsilon}_{N}\left(f_{i}\right)$, defined by

$$
\bar{\epsilon}_{2 N}\left(f_{i}\right)=\frac{\bar{\epsilon}_{N}\left(f_{i}\right)}{2^{\bar{r}_{N}}\left(f_{i}\right)} .
$$

The worst errors $\varepsilon_{N}\left(f_{1}\right)$ and $\varepsilon_{N}\left(f_{2}\right)$ fastly decrease, and $\varepsilon_{N}\left(f_{6}\right)$ is zero, up to rounding errors. This indicates that the quadrature rule $\mathcal{Q}_{N}$ efficiently integrates the smooth functions $f_{1}, f_{2}$, and the symmetric cap function $f_{6}$, independently of the grid orientation. For the function $f_{4}$, which is continuous and non differentiable, the worst error $\varepsilon_{N}\left(f_{4}\right)$ decreases at constant rate. The decrease of the worst error $\varepsilon_{N}\left(f_{5}\right)$ of the "generic" cap function $f_{5}$, which is discontinuous, is slower. And for the spike function $f_{3}$, the worst error $\varepsilon_{N}\left(f_{3}\right)$ slowly decreases, with oscillations.

Roughly speaking, Figure 5 indicates that

$$
\bar{\epsilon}_{N}\left(f_{i}\right) \approx 0.25 \varepsilon_{N}\left(f_{i}\right), i \neq 3, \quad \bar{\epsilon}_{N}\left(f_{3}\right) \approx 0.025 \varepsilon_{N}\left(f_{3}\right) .
$$

Except for $f_{3}$, the worst error is not very large in comparison with the average error (factor 4 ). This indicates that the result is almost insensitive to the grid orientation. For the function $f_{3}$ with a spike, the situation is different (factor 40); the error is sensitive to the grid orientation. Concerning the speed of convergence, we note $\bar{r}_{N}\left(f_{3}\right) \approx 1$ for the spike function, $\bar{r}_{N}\left(f_{5}\right) \approx 1.4$ for the discontinuous cap function, $\bar{r}_{N}\left(f_{4}\right) \approx 2.6$ for the continuous one. The average errors for $f_{1}, f_{2}$ and $f_{6}$ converge fastly, since it was already the case for the worst errors.

\footnotetext{
${ }^{2}$ In order to clarify the figure, we have elimated the following ratios: $\rho_{N}\left(f_{1}\right), N>3$, and $\rho_{N}\left(f_{6}\right)$. Indeed, these ratios are "large", because the associated errors are almost zero.
} 



Figure 5. Statistics of the quadrature error $\epsilon_{N}\left(f_{i}, Q\right)=\left|\int_{\mathbb{S}^{2}} f_{i} \mathrm{~d} \sigma-\mathcal{Q}_{N} f_{i}\left(Q^{\top} \cdot\right)\right|$, where $Q$ scans a set of 1000 random orthogonal matrices. Left: worst error $\varepsilon_{N}\left(f_{i}\right)$. Right: ratio $\rho_{N}\left(f_{i}\right)=\bar{\epsilon}_{N}\left(f_{i}\right) / \varepsilon_{N}\left(f_{i}\right)$ of the average error divided by the worst one.

6.5. Comparison with other quadrature rules. We compare our quadrature rule $\mathcal{Q}_{N}$ with some spherical quadrature rules of the literature, summarized in Table 6

\begin{tabular}{lll}
\hline Abbr. & Description & Ref. \\
\hline CS-BBC21 & Interpolation on the Cubed Sphere by spherical harmonics & This article $\left(\mathcal{Q}_{N}\right)$ \\
CS-CP18 & Octahedral quadrature on the Cubed Sphere, by least-square & $\mid 15$ \\
CS-BC18 & Corrected bivariate trapezoidal on the Cubed Sphere & 1 \\
Lebedev & Gauss quadrature, invariant under the octahedral group & 1 \\
t-design & Spherical $t$-design & 14 \\
\hline
\end{tabular}

TABLE 6. Quadrature of the literature used for comparison.

(1) We use two other rules on the Cubed Sphere; CS-BC18 is a correction of some bivariate trapezoidal rule, CS-CP18 is an octahedral rule which minimizes some least-square error concerning the integration of Legendre spherical harmonics.

(2) We also use "optimal" quadrature rules, whose distribution of nodes is "optimized". Firstly, our rule is invariant under the octahedral group $\mathcal{G}$, so we compare with the Lebedev rule, which is an optimal octahedral rule. Indeed, the optimal grids/weights of Lebedev maximize the degree of precision, under the constraint of invariance under $\mathcal{G}$. Secondly, our weights are quasi-uniform, so we compare with spherical $t$-designs. This rules have equal weights and degree of precision $t$; the associated spherical grids have $\sim \frac{t^{2}}{2}$ nodes, which is the optimal order.

Remark 12. For the Lebedev rules, we have used the Matlab function getLebedevSphere (by R.M. Parrish). The code is available on https://fr.mathworks.com/matlabcentral/fileexchange/27097-getlebedevsphere

The t-designs have been found on R.S. Womersley webpage https://web.maths.unsw.edu.au/〜rsw/Sphere/EffSphDes/sf .html

Results are given on Figure 6. The worst error after 1000 random orthogonal matrices is plotted related to the number of grid points using different quadrature rules. 


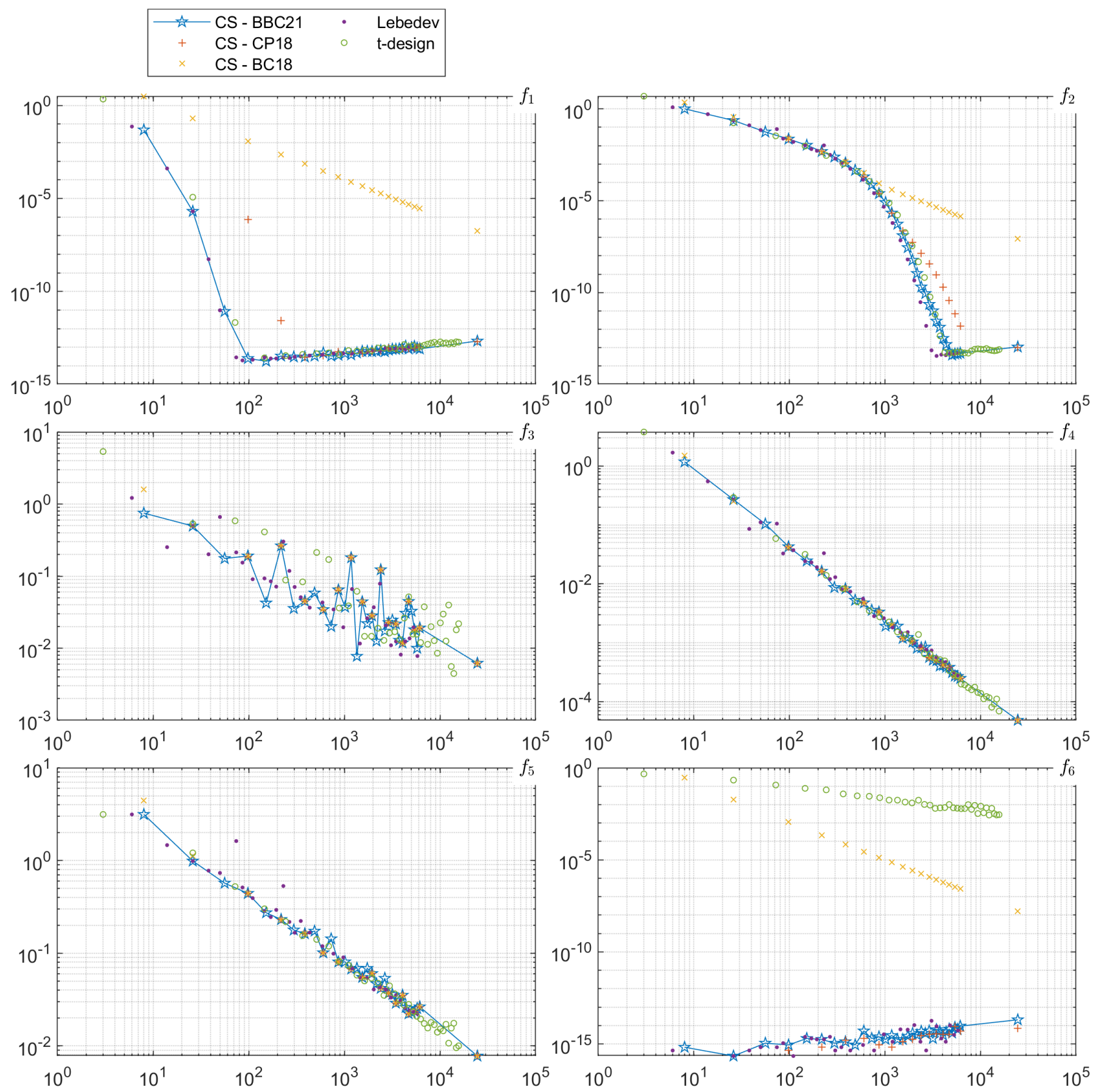

FiguRE 6. Worst quadrature error (for 1000 random orthogonal transformations of the grid), versus the number of grid points.

(1) Among the quadrature rules on $\mathrm{CS}_{N}$, the new rule $\mathcal{Q}_{N}$ outperforms CS-BC18 for the smooth functions $f_{1}$ and $f_{2}$, and for the symmetric cap function $f_{6}$. The rules $\mathcal{Q}_{N}$ and CS-CP18 give similar accuracy for most of the cases, with the following exceptions. The rule $\mathcal{Q}_{N}$ integrates $f_{1}$ more accurately than CS-CP18 before convergence, and $\mathcal{Q}_{N}$ converges slightly faster than CS-CP18 for $f_{2}$.

(2) For the smooth functions $f_{1}$ and $f_{2}$, the rules $\mathcal{Q}_{N}$ and t-design have similar accuracy, whereas the Lebedev rule converges slightly faster. For the function $f_{3}$ with a spike, the worst errors are almost similar; they decay slowly with oscillations. For the cap functions $f_{4}$ and $f_{5}$, the methods converge slowly with similar accuracy. For the "symmetric" cap function $f_{6}, \mathcal{Q}_{N}$ and the Lebedev rule are exact (up to rounding errors) and give better accuracy than the t-design rule.

Overall, the rule $\mathcal{Q}_{N}$ on a fixed grid $\mathrm{CS}_{N}$ displays remarkable accuracy, compared to "optimal" quadrature methods, which require "optimal" grids (Lebedev and t-design rules). 


\section{Conclusion}

Quadrature on the sphere is important in many respects. It arises in a variety of contexts in physics and chemistry: quantum physics, cristallography, gravimetry, astrophysics, kinetic theory (Boltzmann collision kernel approximations, neutron transport), to quote some of them. Our interest in this question stemed from numerical experiments with a Cubed Sphere finite difference (FD) solver for the spherical shallow water equations, [8]. Important mean quantities must be accurately preserved with time, one of them beeing the mean potential vorticity. Since a FD solver is not ab initio conservative, a conservativity analysis must be performed a posteriori, and therefore an accurate quadrature is needed.

The new rule $\mathcal{Q}_{N}$ has the property to be quasi-uniform with positive weights. The numerical results can be compared in accuracy with optimal rules such as t-designs and Lebedev rules. This supports the claim of the "approximation power" of the Cubed Sphere. Among the questions open, a better convergence analysis must be performed. Proving the positivity of the weights is also an important goal. This is deferred to a future study. Overall, the symmetry properties of the Cubed Sphere as a support for quadrature seems a promising topic.

\section{Appendix A. Accuracy of the quadrature rule}

The quadrature rule $\mathcal{Q}_{N}$ is expected to exactly integrate any spherical harmonics of $\mathcal{U}_{N}$, and $15 / 16$ of the Legendre spherial harmonics. In this section, we go further by measuring the accuracy for various tolerances $\epsilon>0$. More precisely, we compute $d_{N}(\epsilon)$, defined as the largest integer such that:

$$
\forall|m| \leq n \leq d_{N}(\epsilon),\left|\int_{\mathbb{S}^{2}} Y_{n}^{m} \mathrm{~d} \sigma-\mathcal{Q}_{N} Y_{n}^{m}\right| \leq \epsilon
$$

The results are reported in Table 7. Except for $N=3,4,64$, the degree $d_{N}\left(10^{-14}\right)$ is $2 N+1$ if $N$ is odd, and $2 N+3$ if $N$ is even. For the particular cases, $d_{N}\left(10^{-14}\right)=4 N-1$ for $N=3$, and $d_{64}\left(10^{-14}\right)=2 \cdot 64+11$.

The table contains implicit information about the default of precision of the first Legendre spherical harmonics that is not well integrated at a fixed accuracy. For instance with $N=8$, the first error which is above $10^{-14}$ is between $10^{-4}$ and $10^{-6}$, and it is reached for the degree 20 (since the method is exact for the odd degree). Such defaults of precision are strong for small values of $N$, but decrease as $N$ grows. For the extreme case $N=1$, there exists a spherical harmonics of degree 4 which is not well integrated, even the tolerance is set to $10^{-4}$. For the extreme case $N=64$, each Legendre function of degree between 139 and 156 is well integrated if the tolerance is $10^{-8}$.

\section{REFERENCES}

[1] C. Ahrens and G. Beylkin. Rotationally invariant quadratures for the sphere. Proceedings of the Royal Society A: Mathematical, Physical and Engineering Sciences, 465(2110):3103-3125, 2009.

[2] C. An and S. Chen. Numerical integration over the unit sphere by using spherical t-design. arXiv:1611.02785v1, 2016.

[3] K. Atkinson and W. Han. Spherical harmonics and approximations on the unit sphere: an introduction, volume 2044. Springer Science \& Business Media, 2012.

[4] C. H. Beentjes. Quadrature on a spherical surface. Technical report, Oxford University ht tps://cbeentjes.github.io/notes/2015-Quadrature-Sphere, 2015.

[5] J.-B. Bellet. Symmetry group of the equiangular cubed sphere. hal-03071135, 2020.

[6] J.-B. Bellet, M. Brachet, and J.-P. Croisille. Interpolation on the Cubed Sphere with Spherical Harmonics. ht tps://hal archives-ouvertes. fr/hal-03202236, 2021.

[7] M. Brachet. Schémas compacts hermitiens sur la Sphère: applications en climatologie et océanographie numérique. PhD thesis, 2018 (in French).

[8] M. Brachet and J.-P. Croisille. Spherical shallow water simulation by a cubed sphere finite difference solver. Quat. J. Roy. Met. Soc., 147(735):786-800, 2021.

[9] J. S. Brauchart and P. J. Grabner. Distributing many points on spheres: Minimal energy and designs. Journal of Complexity, 31(3):293-326, 2015. Oberwolfach 2013.

[10] J. Fliege and U. Maier. The distribution of points on the sphere and corresponding cubature formulae. IMA Journal of Numerical Analysis, 19(2):317-334, 1999.

[11] B. Fornberg and J. M. Martel. On spherical harmonics based numerical quadrature over the surface of a sphere. Advances in Computational Mathematics, 40(5-6):1169-1184, 2014.

[12] K. Hesse, I. H. Sloan, and R. S. Womersley. Numerical integration on the sphere. In W. Freeden, Z. M. Nashed, and T. Sonar, editors, Handbook of Geomathematics. Springer edition, 2010.

[13] V. I. Lebedev. Quadratures on a sphere. USSR Computational Mathematics and Mathematical Physics, 16(2):10-24, 1976.

[14] V. I. Lebedev and D. Laikov. A quadrature formula for the sphere of the 131st algebraic order of accuracy. Doklady Mathematics, 59(3):477-481, 1999.

[15] B. Portelenelle and J.-P. Croisille. An efficient quadrature rule on the cubed sphere. Journal of Computational and Applied Mathematics, 328:59-74, 2018.

[16] C. Ronchi, R. Iacono, and P. S. Paolucci. The "cubed sphere": a new method for the solution of partial differential equations in spherical geometry. Journal of Computational Physics, 124(1):93-114, 1996. 


\begin{tabular}{|c|c|c|c|c|c|c|c|}
\hline$N$ & $\bar{N}$ & $d_{N}\left(10^{-14}\right)$ & $d_{N}\left(10^{-12}\right)$ & $d_{N}\left(10^{-10}\right)$ & $d_{N}\left(10^{-8}\right)$ & $d_{N}\left(10^{-6}\right)$ & $d_{N}\left(10^{-4}\right)$ \\
\hline 1 & 8 & 3 & 3 & 3 & 3 & 3 & 3 \\
\hline 2 & 26 & 7 & 7 & 7 & 7 & 7 & 7 \\
\hline 3 & 56 & 11 & 11 & 11 & 11 & 11 & 11 \\
\hline 4 & 98 & 15 & 15 & 15 & 15 & 15 & 15 \\
\hline 5 & 152 & 11 & 11 & 11 & 11 & 11 & 11 \\
\hline 6 & 218 & 15 & 15 & 15 & 15 & 15 & 17 \\
\hline 7 & 296 & 15 & 15 & 15 & 15 & 15 & 17 \\
\hline 8 & 386 & 19 & 19 & 19 & 19 & 19 & 21 \\
\hline 9 & 488 & 19 & 19 & 19 & 19 & 19 & 23 \\
\hline 10 & 602 & 23 & 23 & 23 & 23 & 23 & 27 \\
\hline 11 & 728 & 23 & 23 & 23 & 23 & 23 & 27 \\
\hline 12 & 866 & 27 & 27 & 27 & 27 & 29 & 33 \\
\hline 13 & 1016 & 27 & 27 & 27 & 27 & 29 & 33 \\
\hline 14 & 1178 & 31 & 31 & 31 & 31 & 33 & 39 \\
\hline 15 & 1352 & 31 & 31 & 31 & 31 & 33 & 41 \\
\hline 16 & 1538 & 35 & 35 & 35 & 35 & 39 & 45 \\
\hline 17 & 1736 & 35 & 35 & 35 & 35 & 39 & 47 \\
\hline 18 & 1946 & 39 & 39 & 39 & 41 & 43 & 51 \\
\hline 19 & 2168 & 39 & 39 & 39 & 41 & 45 & 55 \\
\hline 20 & 2402 & 43 & 43 & 43 & 45 & 49 & 59 \\
\hline 21 & 2648 & 43 & 43 & 43 & 45 & 49 & 65 \\
\hline 22 & 2906 & 47 & 47 & 47 & 49 & 53 & 65 \\
\hline 23 & 3176 & 47 & 47 & 47 & 51 & 55 & 71 \\
\hline 24 & 3458 & 51 & 51 & 51 & 55 & 59 & 75 \\
\hline 25 & 3752 & 51 & 51 & 51 & 55 & 61 & 79 \\
\hline 26 & 4058 & 55 & 55 & 57 & 59 & 65 & 85 \\
\hline 27 & 4376 & 55 & 55 & 57 & 59 & 65 & 89 \\
\hline 28 & 4706 & 59 & 59 & 61 & 65 & 69 & 95 \\
\hline 29 & 5048 & 59 & 59 & 61 & 65 & 71 & 97 \\
\hline 30 & 5402 & 63 & 63 & 65 & 69 & 75 & 101 \\
\hline 31 & 5768 & 63 & 63 & 65 & 69 & 77 & 105 \\
\hline 32 & 6146 & 67 & 67 & 71 & 73 & 81 & 111 \\
\hline 64 & 24578 & 139 & 143 & 147 & 155 & 183 & 255 \\
\hline
\end{tabular}

TABLE 7. Quadrature rule $\mathcal{Q}_{N}$ : observed degree of precision $d_{N}(\epsilon)$ for various tolerances $\epsilon$.

[17] S. L. Sobolev. Cubature formulas on the sphere invariant under finite groups of rotations. Dokl. Akad. Nauk SSSR, 146:310313, 1962.

[18] R. S. Womersley. Spherical designs with close to the minimal number of points. Applied Mathematics Report AMR09/26, Univeristy of New South Wales, Sydney, Austrialia, 2009.

[19] R. S. Womersley. Efficient spherical designs with good geometric properties. In Contemporary computational mathematics-A celebration of the 80th birthday of Ian Sloan, pages 1243-1285. Springer, 2018.

† Université de Lorraine, CNRS, IECL, F-57000 Metz, France

Email address: jean-baptiste.bellet@univ-lorraine.fr, jean-pierre.croisille@univ-lorraine.fr

¥ Université de Poitiers, CNRS, LMA, F-86000 Poitiers, France

Email address: matthieu.brachet@math.univ-poitiers.fr 